\title{
Dosimetric comparison between jaw tracking and static jaw techniques in intensity-modulated radiotherapy
}

\author{
Zhongsu Feng ${ }^{1}$, Hao Wu' ${ }^{2}$ Yibao Zhang ${ }^{2}$, Yunjun Zhang ${ }^{3}$, Jinsheng Cheng ${ }^{1}$ and Xu Su ${ }^{1 *}$
}

\begin{abstract}
Purpose: To compare the dosimetric differences between jaw tracking technique (JTT) and static jaw technique (SJT) in dynamic intensity-modulated radiotherapy (d-IMRT) and assess the potential advantages of jaw tracking technique.
\end{abstract}

Methods: Two techniques, jaw tracking and static jaw, were used respectively to develop the d-IMRT plans for 28 cancer patients with various lesion sites: head and neck, lungs, esophageal, abdominal, prostate, rectal and cervical. The dose volume histograms (DVH) and selected dosimetric indexes for the whole body and for organs at risk (OARs) were compared. A two dimensional ionization chamber Array Seven29 (PTW, Freiburg, Germany) and OCTAVIUS Octagonal phantom (PTW, Freiburg, Germany) were used to verify all the plans.

Results: For all patients, the treatment plans using both techniques met the clinical requirements. The $V_{5}, V_{10}, V_{20}$, $V_{30}, V_{40}$ (volumes receiving $5,10,20,30$ and $40 \mathrm{~Gy}$ at least, respectively), mean dose ( $D_{\text {mean }}$ ) for the whole body and $V_{5}, V_{10}, V_{20}, D_{\text {mean }}$ for lungs in the JT $d$-IMRT plans were significantly less than the corresponding values of the SJT $d$-IMRT plans $(p<0.001)$. The JTT $d$-IMRT plans deposited lower maximum dose $\left(D_{\max }\right)$ to the lens, eyes, brainstem, spinal cord, and right optic nerve, the doses reductions for these OARs ranged from 2.2\% to 28.6\%. The JTT d-IMRT plans deposited significantly lower $D_{\text {mean }}$ to various OARs (all $p$ values $<0.05$ ), the mean doses reductions for these OARs ranged from $1.1 \%$ to $31.0 \%$, and the value reductions depend on the volume and the location of the OARs. The $\gamma$ evaluation method showed an excellent agreement between calculation and measurement for all techniques with criteria of $3 \% / 3 \mathrm{~mm}$.

Conclusions: Both jaw tracking and static jaw d-IMRT plans can achieve comparable target dose coverage. JTT displays superior OARs sparing than SJT plans. These results are of clinical importance, especially for the patients with large and complex targets but close to some highly radio-sensitive organs to spare, and for patients with local recurrent or secondary primary malignant lesion within a previously irradiated area.

Keywords: Jaw tracking, IMRT, Dosimetry, OARs sparing

\section{Introduction}

Nowadays, dynamic intensity-modulated radiotherapy (d-IMRT) is widely used in the treatment of cancer patients. Compared with three dimensional conformal radiotherapy, d-IMRT allows to increase the dose conformity of the target while decreasing normal tissue doses [1].

\footnotetext{
* Correspondence: suxu@nirp.cn

'Chinese Center for Medical Response to Radiation Emergency, National Institute for Radiological Protection, Chinese Center for Disease Control and Prevention, 2 Xinkang Street, Deshengmenwai, Beijing 100088, China
} Full list of author information is available at the end of the article
In dynamic IMRT plans, the multi-leaf collimators (MLCs) translate continuously at variable speeds during the irradiation while the upper and lower jaws stay static. According to the study by LoSasso et al. [2], the MLC transmission increases with increasing jaw field size and beam energy. Cadman et al. [3] found that the transmission through the jaw and the MLC together is smaller than $0.1 \%$. Jaw tracking technique provided by linear accelerators keeps jaws during dose delivery as close as possible to the MLC aperture, and further minimizes leakage and transmission through the MLC leaves. 
Joy et al. [4] evaluated the dosimetric effects of jaw tracking in step-and-shoot IMRT, but failed to indicate which patients would benefit most from jaw tracking. The dosimetric benefits of jaw tracking for prostate and head and neck $(\mathrm{H} \& \mathrm{~N})$ patients using d-IMRT and volumetric-modulated arc therapy (VMAT) were also evaluated by others $[5,6]$. It showed that, the organs far from the target showed larger sparing in jaw-tracking static arc than the organs adjacent to the target. TrueBeam (Varian Medical Systems, Palo Alto, CA) is a new generation of linear accelerator providing jaw tracking technique (JTT), which traces the MLC aperture with jaws to minimize the leakage and transmission of the MLC leaves, hence further reduces the OAR doses adjacent to the target, and potentially improves the dose falloff towards the surrounding critical structures.

The main purpose of this study is to assess the potential advantages of the JTT provided by TrueBeam accelerators in reducing the doses to the organs at risk (OARs) while preserving adequate target dose coverage, and to evaluate which patients and which OARs would benefit most from JTT plans.

\section{Materials and methods}

\section{Ethical consideration and consent}

The use of the radiotherapy database for retrospective research has been approved by the committee of the Ethical Review Board of the Chinese Center for Disease Control and Prevention (No. LLSC2014-005). This research was waivered informed consent.

\section{Patient characteristics}

Twenty eight cancer patients (median age 58 years, range 31-77 years) treated with radiotherapy in our department were retrospectively included in this study. The lesion distributions of the selected cases included head and neck, lungs, esophageal, abdominal, prostate, rectal and cervical, as shown in Table 1. One local recurrent nasopharyngeal carcinoma (NPC) patient was included in the H\&N group.

\section{Contouring of targets and OARs}

All patients were immobilized with custom-made thermoplastic mask and underwent computed tomography (CT) scan with slice thickness of $3 \mathrm{~mm}$ (for head and neck patients) or $5 \mathrm{~mm}$ (for thoracic, abdominal and pelvic patients ) using the Siemens Somatom Sensation Open 40 slice CT scanner (Siemens, Forchheim, Germany). The targets and OARs were contoured slice by slice on the treatment planning CT images. Relevant organs were delineated for different anatomic regions. The body was segmented automatically by the Eclipse (Versions 11.0) Treatment Planning System (TPS) (Varian Medical Systems, Palo Alto, CA). Isotropic margins of $3 \mathrm{~mm}$ (for head and neck patients) or $5 \mathrm{~mm}$ (for thoracic, abdomen and pelvic patients) were created around the clinical target volume (CTV) and gross tumor volume (GTV) to generate the planning target volume (PTV) and planning gross tumor volume (PGTV), respectively.

\section{Treatment planning}

The static jaw technique (SJT) d-IMRT plans were created using the Eclipse TPS using sliding window dynamic delivery and fixed beam angles. The selected energies were $6 \mathrm{MV}$ (for head and neck patients) and $10 \mathrm{MV}$ (for thoracic, abdominal and pelvic patients) respectively from a TrueBeam linear accelerator. The collimator angles were optimized according to the target shape. After IMRT optimization using the dose volume optimizer algorithm (version 11.0.31), the volumetric doses were calculated using the anisotropic analytical algorithm (version 11.0.31) with a dose calculation grid of $2 \mathrm{~mm}$. The reference volume for the treatment planning was PTV. The normal tissue dose volume constraints used for the treatment planning are listed in Table 2. For all patients, the dose distributions met the planning requirements, i.e. at least $95 \%$ of the PTV received the prescribed dose, while the doses to the surrounding tissues were minimized. To create JTT d-IMRT plans, the SJT d-IMRT plans were duplicated and jaw tracking function was selected when calculating the leaf motions and volumetric doses. All the machine parameters and optimization parameters were

Table 1 Lesion distributions and treatment characteristics of the selected cases

\begin{tabular}{|c|c|c|c|c|c|}
\hline \multirow[t]{3}{*}{ Lesion sites } & \multirow[t]{3}{*}{$\mathrm{n}$} & \multicolumn{2}{|l|}{ Total dose (Gy) } & \multicolumn{2}{|c|}{ PTV $\left(\mathrm{cm}^{3}\right)$} \\
\hline & & GTV & CTV & & \\
\hline & & Mean dose (range) & Mean dose (range) & Mean & SD \\
\hline Head and neck & 6 & $66.4(60-70)$ & $55.8(45-60)$ & 693.38 & 168.8 \\
\hline Lungs & 5 & $60.7(56-66)$ & $54.9(50.6-60)$ & 270 & 50.07 \\
\hline Esophageal & 4 & $65(64,66)$ & 50 & 536.28 & 39.4 \\
\hline Abdominal & 3 & $53.5(50-60)$ & $46(45-48)$ & 454.1 & 181.24 \\
\hline Prostate & 2 & 72 & $65(60,70)$ & 174.85 & 35.75 \\
\hline Rectal & 6 & 50.6 & 41.8 & 1163.57 & 55.14 \\
\hline Cervical & 2 & - & $47.5(45,50)$ & 1204.05 & 139.45 \\
\hline
\end{tabular}


Table 2 Dose constraints used in treatment planning

\begin{tabular}{|c|c|c|c|}
\hline Organ & Dose volume parameters & Organ & Dose volume parameters \\
\hline Lens & $D_{\max }<7 G y$ & Heart & $\mathrm{D}_{\text {mean }}<26 \mathrm{~Gy}$ \\
\hline \multirow[t]{2}{*}{ Eye } & $\mathrm{D}_{\max }<50 \mathrm{~Gy}$ & & $V_{30}<50 \%$ \\
\hline & $\mathrm{D}_{\text {mean }}<35 \mathrm{~Gy}$ & Liver & $\mathrm{D}_{\text {mean }}<30 \mathrm{~Gy}$ \\
\hline Optic nerve & $\mathrm{D}_{\max }<54 \mathrm{~Gy}$ & & $1 / 3$ volume of liver avoid irradiation \\
\hline Brain stem & $\mathrm{D}_{\max }<54 \mathrm{~Gy}$ & Kidney & $\mathrm{D}_{\text {mean }}<18 \mathrm{~Gy}$ \\
\hline \multirow[t]{2}{*}{ Parotid } & $V_{30}<50 \%$ & & $V_{12}<55 \%$ \\
\hline & $D_{\text {mean }}<26 G y$ & & $V_{20}<32 \%$ \\
\hline Spinal cord & $D_{\max }<40 G y$ & Intestine & $V_{40}<30 \%$ \\
\hline \multirow[t]{2}{*}{ Esophagus } & $V_{35}<50 \%$ & Bladder & $V_{30}<50 \%$ \\
\hline & $\mathrm{D}_{\text {mean }}<34 \mathrm{~Gy}$ & Femoral Head & $V_{20}<50 \%$ \\
\hline \multirow[t]{4}{*}{ Lung } & $V_{5}<70 \%$ & & $V_{30}<15 \%$ \\
\hline & $V_{10}<50 \%$ & Rectum & $V_{30}<50 \%$ \\
\hline & $V_{20}<30 \%$ & & $V_{60}<35 \%$ \\
\hline & $D_{\text {mean }}<15 G y$ & & $V_{70}<20 \%$ \\
\hline
\end{tabular}

identical to those of the SJT d-IMRT plans. The JTT plans reduce the dose leakage and transmission through the MLCs, which required renormalization to achieve the same PTV coverage as in the SJT d-IMRT plans.

\section{Plan evaluation}

The DVHs and the isodose curves of the JTT plans were compared with that of the SJT plans. The dose changes to the whole body were evaluated using $V_{5}, V_{10}, V_{20}$, $\mathrm{V}_{30}, \mathrm{~V}_{40}, \mathrm{D}_{\max }$ and $\mathrm{D}_{\text {mean }}$ as listed in Table 3 . The parameters of $V_{5}, V_{10}, V_{20}, D_{\max }$, and $D_{\text {mean }}$ were used to evaluate the dose changes to the lungs, as shown in Table 4. The dosimetric disparities of OARs were evaluated using the maximum dose $\left(D_{\max }\right)$ and mean dose $\left(\mathrm{D}_{\text {mean }}\right)$ as listed in Table 5 and Table 6.

\section{Plan verification}

The Eclipse TPS was used to create plans for verification by converting the SJT and JTT plans to OCTAVIUS Octagonal phantoms (PTW, Freiburg, Germany). Then the volumetric doses were re-calculated, and the dose

Table 3 Comparison of the whole body doses between the two techniques $(\bar{X} \pm s)$

\begin{tabular}{llll}
\hline Body & SJT & JTT & p \\
\hline$V_{5}(\%)$ & $37.2 \pm 19.1$ & $36.1 \pm 18.6$ & $<0.001$ \\
$V_{10}(\%)$ & $29.0 \pm 16.5$ & $28.2 \pm 16.0$ & $<0.001$ \\
$V_{20}(\%)$ & $19.9 \pm 12.9$ & $19.3 \pm 12.4$ & $<0.001$ \\
$V_{30}(\%)$ & $12.0 \pm 9.6$ & $11.6 \pm 9.2$ & $<0.001$ \\
$V_{40}(\%)$ & $7.6 \pm 7.0$ & $7.5 \pm 6.9$ & $<0.001$ \\
$D_{\text {max }}(G y)$ & $60.3 \pm 8.5$ & $60.5 \pm 8.8$ & 0.071 \\
$D_{\text {mean }}(G y)$ & $9.8 \pm 6.0$ & $9.6 \pm 5.8$ & $<0.001$ \\
\hline
\end{tabular}

distributions in effective measurement points of the two dimensional ionization chamber Array Seven29 (PTW, Freiburg, Germany) were exported for all plans. By using the $\gamma$ evaluation method of VeriSoft software (version 5.1) (PTW, Freiburg, Germany), the measured dose planes were compared with the computed dose distribution using criteria of $3 \%$ dose difference and $3 \mathrm{~mm}$ distance to agreement.

\section{Statistical analysis}

The data were presented as the averages of all patients followed by the standard deviation $(\bar{X} \pm s)$. The PASW Statistics (version 18.0) software (SPSS; Chicago, IL, USA) was applied for statistical analysis. To compare the results of the two techniques, Wilcoxon Signed-Ranks test was performed with $\mathrm{p}$ values $<0.05$ considered as significant.

\section{Results}

As shown in the DVH comparison (Figure 1), JTT dIMRT plans reduce doses to the normal tissues, especially in the low dose regions.

Table 4 Comparison of lung doses between the two techniques $(\bar{X} \pm s)$

\begin{tabular}{llll}
\hline Lung & SJT & JTT & $\mathbf{p}$ \\
\hline$V_{5}(\%)$ & $48.3 \pm 6.7$ & $45.7 \pm 5.9$ & $<0.001$ \\
$V_{10}(\%)$ & $34.9 \pm 6.2$ & $33.6 \pm 5.8$ & $<0.001$ \\
$V_{20}(\%)$ & $20.5 \pm 5.5$ & $19.9 \pm 5.1$ & $<0.001$ \\
$D_{\text {max }}(G y)$ & $59.9 \pm 6.4$ & $60.00 \pm 6.5$ & 0.155 \\
$D_{\text {mean }}(G y)$ & $11.4 \pm 2.6$ & $11.0 \pm 2.5$ & $<0.001$ \\
\hline
\end{tabular}


Table 5 Comparison of OARs $D_{\max }$ between the two techniques $(\bar{X} \pm s)$

\begin{tabular}{llll}
\hline OARs $\mathbf{D}_{\max }(\mathrm{Gy})$ & SJT & JTT & $\mathbf{p}$ \\
\hline Right lens & $5.6 \pm 2.2$ & $4.00 \pm 1.7$ & 0.031 \\
Left lens & $5.7 \pm 1.6$ & $4.2 \pm 1.3$ & 0.031 \\
Right eye & $26.3 \pm 17.3$ & $24.5 \pm 17.8$ & 0.031 \\
Left eye & $26.6 \pm 13.9$ & $24.9 \pm 14.9$ & 0.031 \\
Right optic nerve & $44.3 \pm 8.9$ & $43.4 \pm 8.7$ & 0.031 \\
Left optic nerve & $41.8 \pm 10.8$ & $40.8 \pm 12.0$ & 0.156 \\
Brain stem & $39.1 \pm 12.0$ & $37.2 \pm 12.9$ & 0.016 \\
Right parotid & $62.8 \pm 10.9$ & $63.2 \pm 11.4$ & 0.063 \\
Left parotid & $63.2 \pm 7.3$ & $63.6 \pm 7.4$ & 0.156 \\
Spinal cord & $31.0 \pm 8.0$ & $30.3 \pm 7.9$ & $<0.001$ \\
Esophagus & $62.9 \pm 5.5$ & $62.8 \pm 5.5$ & 0.500 \\
Heart & $57.9 \pm 5.9$ & $57.9 \pm 6.0$ & 0.527 \\
Liver & $49.2 \pm 6.1$ & $49.2 \pm 6.1$ & 0.094 \\
Right kidney & $36.3 \pm 15.9$ & $36.3 \pm 16.0$ & 0.406 \\
Left kidney & $37.2 \pm 9.3$ & $37.0 \pm 9.1$ & 0.406 \\
Intestine & $50.6 \pm 3.7$ & $50.7 \pm 3.9$ & 0.313 \\
Bladder & $56.1 \pm 9.0$ & $56.2 \pm 8.9$ & 0.156 \\
Femoral head & $39.7 \pm 5.2$ & $39.8 \pm 5.3$ & 0.156 \\
Rectum & $60.6 \pm 8.9$ & $60.2 \pm 9.4$ & 0.156 \\
\hline
\end{tabular}

Table 6 Comparison of OARs $D_{\text {mean }}$ between the two techniques $(\bar{X} \pm s)$

\begin{tabular}{llll}
\hline OARs $\mathbf{D}_{\text {mean }}$ (Gy) & SJT & JTT & $\mathbf{p}$ \\
\hline Right lens & $4.9 \pm 2.0$ & $3.38 \pm 1.44$ & 0.031 \\
Left lens & $5.0 \pm 1.4$ & $3.59 \pm 1.07$ & 0.031 \\
Right eye & $7.1 \pm 3.8$ & $5.56 \pm 3.55$ & 0.031 \\
Left Eye & $7.7 \pm 2.6$ & $5.99 \pm 2.83$ & 0.031 \\
Right optic nerve & $25.5 \pm 12.4$ & $23.87 \pm 13.33$ & 0.031 \\
Left optic nerve & $26.0 \pm 11.1$ & $24.37 \pm 12.26$ & 0.031 \\
Brain stem & $23.7 \pm 13.5$ & $22.03 \pm 14.08$ & 0.016 \\
Right parotid & $29.3 \pm 4.9$ & $27.41 \pm 4.02$ & 0.031 \\
Left parotid & $28.7 \pm 4.1$ & $27.23 \pm 3.46$ & 0.031 \\
Spinal cord & $19.0 \pm 8.0$ & $18.1 \pm 7.6$ & $<0.001$ \\
Esophagus & $29.4 \pm 8.4$ & $28.9 \pm 8.1$ & 0.016 \\
Heart & $17.9 \pm 11.9$ & $17.5 \pm 11.8$ & 0.004 \\
Liver & $12.2 \pm 9.9$ & $11.9 \pm 9.8$ & 0.031 \\
Right kidney & $8.5 \pm 4.1$ & $8.2 \pm 4.0$ & 0.031 \\
Left kidney & $6.6 \pm 4.8$ & $6.3 \pm 4.5$ & 0.031 \\
Intestine & $27.4 \pm 3.4$ & $27.1 \pm 3.4$ & 0.016 \\
Bladder & $33.5 \pm 8.7$ & $33.1 \pm 8.5$ & 0.004 \\
Femoral head & $14.1 \pm 1.6$ & $13.7 \pm 1.5$ & 0.004 \\
Rectum & $35.8 \pm 4.5$ & $35.2 \pm 4.7$ & 0.031 \\
\hline
\end{tabular}

Table 3 lists the comparison of the whole body doses between the two techniques. The JTT d-IMRT plans displayed significantly lower percentages of $V_{5}, V_{10}, V_{20}$, $\mathrm{V}_{30}, \mathrm{~V}_{40}$ and significantly lower mean doses of the whole body $(p<0.001)$. There was no significant difference of maximum doses delivered to the whole body $(p=0.071)$.

Table 4 lists the statistical data of the lungs for all thoracic cases. The $\mathrm{V}_{5}, \mathrm{~V}_{10}, \mathrm{~V}_{20}, \mathrm{D}_{\text {mean }}$ of lungs in JTT d-IMRT plans are significantly lower than the corresponding values of the SJT d-IMRT plans $(\mathrm{p}<0.001)$, the mean reduction was $2.6 \%, 1.3 \%, 0.6 \%$ and $0.4 \%$, respectively. There was no significant difference of maximum doses delivered to the lungs $(\mathrm{p}=0.155)$.

This study demonstrates that the mean values of the maximum doses to the lens, eyes, brainstem, spinal cord, and right optic nerve are significantly reduced in the JTT plans (all p values $<0.05$ ), as shown in Table 5 . The doses reduction for these OARs ranged from $2.2 \%$ to $28.6 \%$, and the maximum dose to lens is reduced most obviously.

Table 6 lists the mean doses to various OARs for all patients. The JTT d-IMRT plans give significantly lower mean doses to the studied OARs (all p values <0.05). The mean doses reduction for these OARs ranged from $1.1 \%$ to $31.0 \%$, and the reduction values are depend on the volume and the location of the OARs.

Treatment plan verification of the two techniques is generally performed with the $\gamma$ evaluation method. The $\gamma$ was compared locally, and the $\gamma$ pass rate for SJT and JTT is $93.9 \pm 1.98$ and $94.1 \pm 1.95$, respectively. The comparison of $\gamma$ between the two techniques showed no statistical difference $(\mathrm{p}=0.132)$. Dose calculation and delivery are both accurate for SJT and JTT plans at criteria of $3 \% / 3 \mathrm{~mm}$ using $\gamma$ analysis.

\section{Discussion}

Jaw tracking methods have also been used in other treatment modalities aiming to achieve better target coverage and critical structure sparing. Joy et al. [4] evaluated the dosimetric effects of jaw tracking in step-and-shoot IMRT, showing an overall reduction of normal tissue doses. Most patients had reductions of $\mathrm{V}_{5}, \mathrm{~V}_{10}$, and $\mathrm{V}_{20}$ by less than $2 \%$ in the normal tissues. Schmidhalter et al. [5] evaluated the leaf transmission reduction using moving jaws in dynamic MLC IMRT, and demonstrated that the undesired doses to the body volume minus the planning target volume decreased by up to $1.8 \%$ and $1.5 \%$ for prostate and $\mathrm{H} \& N$ patients. Simultaneously, the MU increased by up to $3.1 \%$ and $2.8 \%$, respectively. Kim et al. [6] assessed the potential advantages of jaw tracking technique by using control point sequences of VMAT planning, showing that, for $\mathrm{H} \& \mathrm{~N}$ cases, the mean dose reductions for all the OARs ranged from $4.3 \%$ to $11.9 \%$, and for prostate patients, the organs distant from the target were spared better in jaw- 

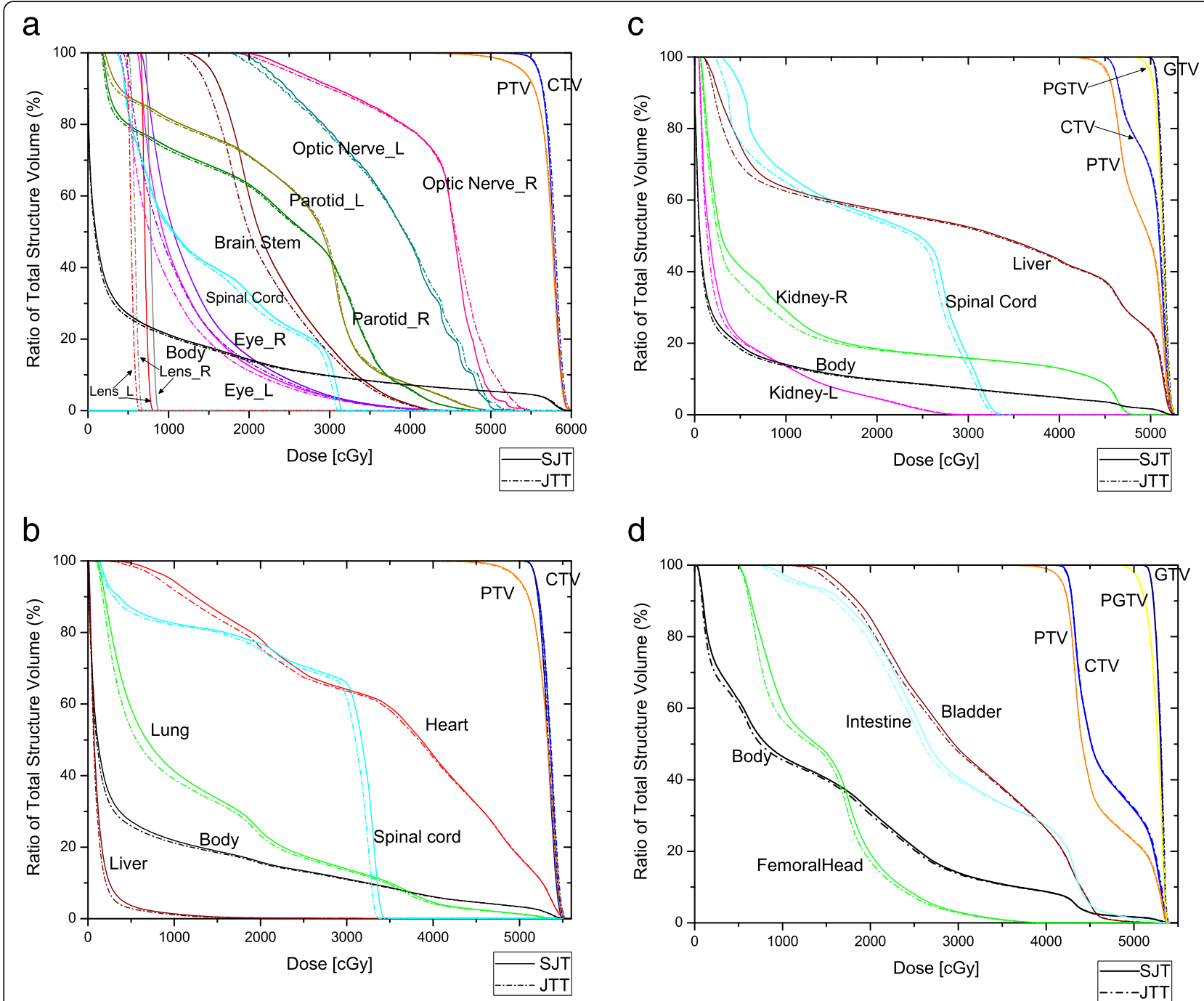

d

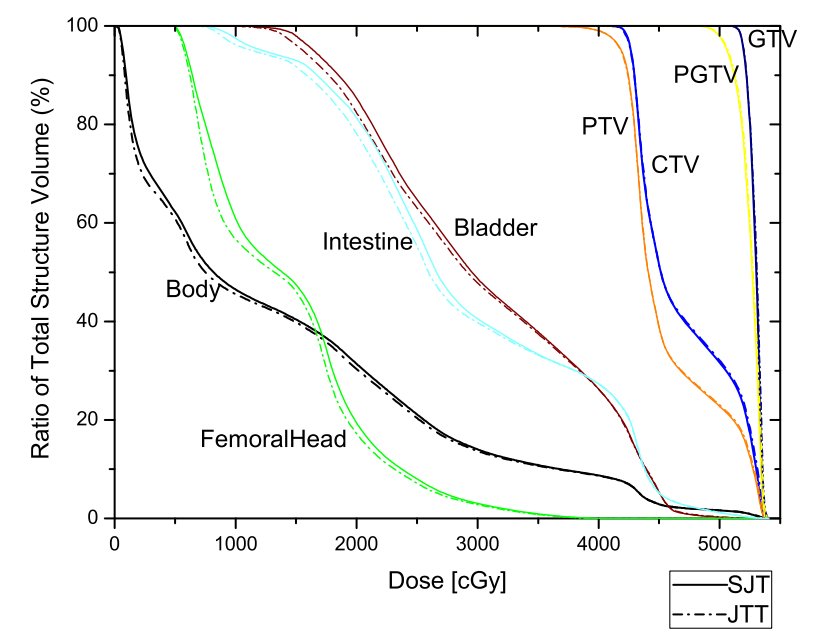

Figure 1 DVH comparison between SJT and JTT plans for different body sites: (a) H\&N, (b) Thoracic, (c) Abdominal, (d) Pelvic case.

CTV: Clinical target volume; PTV: Planning target volume; GTV: Gross tumor volume; PGTV: Planning gross tumor volume.

tracking static arc (JTSA) plans. The dose reductions were more significant in the dose regions of $\mathrm{D}_{80}, \mathrm{D}_{90}$ and $\mathrm{D}_{95}$ for all the patients in JTSA plans.

However, previous studies [4-6] included no abdominal cases or other pelvic lesion except prostate: the number of OARs was less than that of this study. Moreover, they didn't indicate which cases and which organs would benefit most from JTT.

This study selected cases of various body sites. For large and complex targets, such as NPC, the large target volumes are surrounded by many critical neural tissues and sensitive structures such as lens, optic nerves, brain stem, parotid glands and spinal cord. This study demonstrates that the mean values of the maximum doses to the lens, eyes, brainstem, spinal cord, and right optic nerve are significantly reduced in the JTT plans (all $\mathrm{p}$ values $<0.05)$ as shown in Table 5 . Perhaps because these OARs do not overlap with the target in the selected cases, yet the left optic nerve and other OARs are partially or wholly covered by PTVs. The reduction of the maximum dose to OARs may reduce the risk of radiation injury $[7,8]$, such as radiation induced myelitis and cataract. It is also more meaningful for the radiotherapy patients with local recurrent or second primary malignant lesion within or adjacent to a previously irradiated area.

The JTT technique also significantly reduces the low dose regions. In the thoracic cases, the JTT technique significantly reduces the $\mathrm{V}_{5}, \mathrm{~V}_{10}, \mathrm{~V}_{20}$ to the lungs, as shown in Table 4, compared to the SJT plan $(\mathrm{p}<0.001)$. The results may mostly benefit Hodgkin's lymphoma patients whose lungs are largely within the treatment 
fields during radiotherapy. The JTT technique may reduce the risk of pulmonary toxicity since radiation pneumonitis rate is correlated with low dose volumes [9-12]. The risk of radiation induced secondary malignancies is another concern, which is strongly associated with low dose exposure of normal tissues during IMRT [13].

In addition, this study suggests that the JTT plans give significantly lower mean doses to various OARs (all $\mathrm{p}$ values $<0.05$, refer to Table 6 for details). The JTT associated OAR dose reductions are more obvious for the mean doses to the structures of small volume (such as lens) or structures far from the targets (such as left optic nerve, esophagus, and kidneys in this study). The mean doses of OARs have been widely used to predict the probability of radiation toxicity [9-12], such as pulmonary toxicity. Therefore, JTT plans may decrease the probability of developing late side effects or secondary neoplasm.

Some problems exist in the current jaw tracking technique though. Firstly, jaw tracking is not considered in the optimization of JTT d-IMRT or in the step-andshoot jaw tracking IMRT plans [4], as limited by the optimization algorithm of TPS. However, the Eclipse TPS (version 11.0) provides jaw tracking option in the VAMT plan optimization, which is applicable on Varian Truebeam accelerators. Secondly, the output factor of the accelerator varies dramatically in jaw tracking technique. Usually, the output factors are measured on the central axis of the beam. However, there are many offaxis fields in jaw tracking plans, which would affect the output factor and should be considered in treatment planning. Joy et al. [4] calculated the off-axis output factors and demonstrated decreases of $1 \%$ to $3 \%$ when shifting away from central axis. Although all the SJT and JTT plans in this study can pass the dosimetric verification, this issue was not solved so far and further research is needed. Thirdly, the performance of flattening filter-free (FFF) module combined with the jaw tracking technique of TrueBeam accelerator is still uncertain. There were several reports [14-19] about the clinical usage of FFF beams, indicating more potential of reducing the doses to OARs with comparable target dose coverage. The potential benefit of applying jaw tracking technique in FFF beams will be further studied in the future work.

\section{Conclusions}

This study demonstrates that all the SJT and JTT plans could meet the clinical objectives and are clinically applicable. The dose measurements agreed well with TPS calculated doses. JTT displays superior OARs sparing than SJT plans. These results are of clinical importance, especially for the patients with large and complex targets but close to some highly radio-sensitive organs to spare (such as lens or gonad), and for patients with local recurrent or secondary primary malignant lesion within a previously irradiated area. More studies on off-axis output factors and combined application of FFF module should be conducted in the future.

\section{Abbreviations}

JTT: Jaw tracking technique; SJT: Static jaw technique; d-IMRT: dynamic Intensity-modulated radiotherapy; DVH: Dose-volume histogram; OARs: Organs at risk; $V_{x}$ : Percentage volume of organs receiving at least the $x G y$; MLC: Multi-leaf collimator; VMAT: Volumetric-modulated arc therapy; H\&N: Head and neck; NPC: Nasopharyngeal carcinoma; CT: Computed tomography; TPS: Treatment planning system; CTV: Clinical target volume; PTV: Planning target volume; GTV: Gross tumor volume; PGTV: Planning gross tumor volume; SD: standard deviation; JTSA: Jaw-tracking static arc; LMC: Leaf motion calculator; FFF: Flattening filter-free.

\section{Competing interests}

The authors declare that they have no competing interests.

\section{Authors' contributions}

ZSF contributed to the data acquisition, statistical testing, data analysis, and manuscript drafting. HW and XS are responsible for the design of the study and have also critically revised the manuscript. YBZ and JSC have contributed to the analyses and interpretation of the results and have critically revised the manuscript. YJZ has contributed to the statistical analyses. All authors provided approval of the final manuscript.

\section{Research support}

This work was supported by the specialized project on scientific research within healthcare circle by National Health and Family Planning Commission of China (201002009).

\section{Author details}

'Chinese Center for Medical Response to Radiation Emergency, National Institute for Radiological Protection, Chinese Center for Disease Control and Prevention, 2 Xinkang Street, Deshengmenwai, Beijing 100088, China. ${ }^{2}$ Key laboratory of Carcinogenesis and Translational Research (Ministry of Education), Department of Radiotherapy, Peking University Cancer Hospital \& Institute, 52 Fuchen Road, Haidian, Beijing 100142, China. ${ }^{3}$ School of Foundational Education, Peking University Health Science Center, 38 Xueyuan Road, Haidian, Beijing 100191, China.

Received: 12 August 2014 Accepted: 7 January 2015

Published online: 27 January 2015

\section{References}

1. Verhey L. Comparison of three-dimensional conformal radiation therapy and intensity-modulated radiation therapy systems. Semin Radiat Oncol. 1999:9(1):78-98.

2. LoSasso T, Chui CS, Ling CC. Physical and dosimetric aspects of a multileaf collimation system used in the dynamic mode for implementing intensity modulated radiotherapy. Med Phys. 1998;25(10):1919-27.

3. Cadman P, McNutt T, Bzdusek K. Validation of physics improvements for IMRT with a commercial treatment-planning system. J Appl Clin Med Phys. 2005;6:74-86.

4. Joy S, Starkschall G, Kry S, Salehpour M, White RA, Lin SH, et al. Dosimetric effects of jaw tracking in step-and-shoot intensity-modulated radiation therapy. J Appl Clin Med Phys. 2012;13(2):136-45.

5. Schmidhalter D, Fix MK, Niederer P, Mini R, Manser P. Leaf transmission reduction using moving jaws for dynamic MLC IMRT. Med Phys. 2007;34(9):3674-87.

6. Kim JI, Park JM, Park SY, Choi CH, Wu HG, Ye SJ. Assessment of potential jaw-tracking advantage using control point sequences of VMAT planning. J Appl Clin Med Phys. 2014;15:160-8.

7. Bragg CM, Conway J, Robinson MH. The role of intensity-modulated radiotherapy in the treatment of parotid tumors. Int J Radiat Oncol Biol Phys. 2002;52(3):729-38. 
8. Emami B, Lyman J, Brown A, Coia L, Goitein M, Munzenrider JE, et al. Tolerance of normal tissue to therapeutic irradiation. Int J Radiat Oncol Biol Phys. 1991;21(1):109-22.

9. Bradley JD, Hope A, El Naqa I, Apte A, Lindsay PE, Bosch W, et al. A nomogram to predict radiation pneumonitis, derived from a combined analysis of RTOG 9311 and institutional data. Int J Radiat Oncol Biol Phys. 2007;69(4):985-92.

10. Wang S, Liao Z, Wei X, Liu HH, Tucker SL, Hu CS, et al. Analysis of clinical and dosimetric factors associated with treatment-related pneumonitis (TRP) in patients with non-small-cell lung cancer (NSCLC) treated with concurrent chemotherapy and three-dimensional conformal radiotherapy (3D-CRT). Int J Radiat Oncol Biol Phys. 2006;66(5):1399-407.

11. Tucker SL, Liu HH, Wang S, Wei X, Liao Z, Komaki R, et al. Dose-volume modeling of the risk of postoperative pulmonary complications among esophageal cancer patients treated with concurrent chemoradiotherapy followed by surgery. Int J Radiat Oncol Biol Phys. 2006;66(3):754-61.

12. Kong FM, Hayman JA, Griffith KA, Kalemkerian GP, Arenberg D, Lyons S, et al. Final toxicity results of a radiation-dose escalation study in patients with non-small-cell lung cancer (NSCLC): predictors for radiation pneumonitis and fibrosis. Int J Radiat Oncol Biol Phys. 2006;65(4):1075-86.

13. Chera BS, Rodriguez C, Morris CG, Louis D, Yeung D, Li Z, et al. Dosimetric comparison of three different involved nodal irradiation techniques for stage II Hodgkin's lymphoma patients: conventional radiotherapy, intensity-modulated radiotherapy, and three-dimensional proton radiotherapy. Int J Radiat Oncol Biol Phys. 2009;75:1173-80.

14. Zhuang M, Zhang T, Chen Z, Lin Z, Li D, Peng X, et al. Advanced nasopharyngeal carcinoma radiotherapy with volumetric modulated arcs and the potential role of flattening filter-free beams. Radiat Oncol. 2013:8:120.

15. Nicolini G, Ghosh-Laskar S, Shrivastava SK, Banerjee S, Chaudhary S, Agarwal JP, et al. Volumetric modulation arc radiotherapy with flattening filter-free beams compared with static gantry IMRT and 3D conformal radiotherapy for advanced esophageal cancer: a feasibility study. Int J Radiat Oncol Biol Phys. 2012;84:553-60.

16. Subramaniam S, Thirumalaiswamy S, Srinivas C, Gandhi GA, Kathirvel M, Kumar KK, et al. Chest wall radiotherapy with volumetric modulated arcs and the potential role of flattening filter free photon beams. Strahlenther Onkol. 2012;188:484-91.

17. Scorsetti M, Alongi F, Castiglioni S, Clivio A, Fogliata A, Lobefalo F, et al. Feasibility and early clinical assessment of flattening filter free (FFF) based stereotactic body radiotherapy (SBRT) treatments. Radiat Oncol. 2011;6:113

18. Georg D, Knöös T, McClean B. Current status and future perspective of flattening filter free photon beams. Med Phys. 2011;38:1280-93.

19. Cashmore J, Ramtohul M, Ford D. Lowering whole-body radiation doses in pediatric intensity-modulated radiotherapy through the use of unflattened photon beams. Int J Radiat Oncol Biol Phys. 2011;80(4):1220-7.

\section{Submit your next manuscript to BioMed Central and take full advantage of:}

- Convenient online submission

- Thorough peer review

- No space constraints or color figure charges

- Immediate publication on acceptance

- Inclusion in PubMed, CAS, Scopus and Google Scholar

- Research which is freely available for redistribution 